\title{
Diabetes and the occurrence of ventricular arrhythmic events in patients with severe left ventricular dysfunction
}

\author{
D. Aronson ${ }^{1}$, A. J. Burger ${ }^{2}$ \\ ${ }^{1}$ Beth Israel Deaconess Medical Centre, Harvard Medical School, Boston, Mass., USA \\ ${ }^{2}$ Cardiology Division, RAMBAM Medical Centre, Haifa, Israel
}

\section{Abstract}

Aims/hypothesis. Diabetes is associated with a higher risk of ischaemic heart disease and heart failure, which frequently lead to rhythm disorders. However, experimental and clinical studies are inconsistent with regard to the risk of arrhythmic events in patients with diabetes. We studied the frequency of ventricular arrhythmic events in patients with and without diabetes. Methods. The study group included 207 patients with severe heart failure. The severity of ventricular arrhythmias was assessed by 24-h Holter monitoring, using prospectively defined measures of ventricular arrhythmic burden.

Results. Patients with diabetes comprised $48 \%$ of the study group. All measures of ventricular ectopy were much lower in patients with diabetes. Mean hourly ventricular pairs $(13.3 \pm 3.7$ vs $7.2 \pm 2.0, p=0.03)$, mean hourly repetitive ventricular beats $(32 \pm 9$ vs $17 \pm 5$, $p=0.01$ ), and the frequency of ventricular tachycardia episodes per $24 \mathrm{~h}(36 \pm 13$ vs $16 \pm 6, p=0.01)$ were lower in patients with diabetes. After inclusion of clinical variables and drug therapies in a multivariate analysis, the negative relationship between diabetes and the frequency of ventricular pairs $(p=0.03)$, repetitive ventricular beats $(p=0.04)$, and ventricular tachycardia $(p=0.03)$ remained independent. In multivariate logistic regression, the risk of developing ventricular tachycardia was lower in patients with diabetes with an adjusted odds ratio of 0.41 (95\% CI, 0.22 to 0.75 , $p=0.004$ ).

Conclusion/interpretation. Diabetes confers a protective effect with regard to the occurrence of ventricular arrhythmias in the setting of decompensated heart failure. The precise mechanism of this phenomenon requires further investigation. [Diabetologia (2002) 45:1440-1445]

Keywords Cardiomyopathy, heart failure, diabetes mellitus, sudden cardiac death, ventricular arrhythmias.
Diabetes is a powerful and independent risk factor for cardiovascular mortality and morbidity, accounting for virtually $80 \%$ of all deaths among North American patients with diabetes $[1,2]$. Several studies have sug-

Received: 13 March 2002 / Revised: 2 May 2002

Published online: 16 August 2002

C) Springer-Verlag 2002

Corresponding author: A. J. Burger, MD, Beth Israel Deaconess Medical Center, West Campus, Non-invasive Cardiology Laboratory, Baker-3, 1 Deaconess Road, Boston, Mass. 02215, USA. E-mail: aburger@ caregroup.harvard.edu

Abbreviations: CHF, Congestive heart failure; PAC, premature atrial beat; PVC, premature ventricular beats. gested that increased susceptibility for ventricular arrhythmias and sudden cardiac death contribute to the increased cardiovascular mortality in patients with diabetes [3]. In addition, autonomic neuropathy, a common complication of diabetes [4], has been frequently postulated to increase susceptibility to ventricular arrhythmias and sudden death $[5,6]$. The underlying hypothesis is that fatal ventricular arrhythmias could be precipitated by a disturbance of autonomic balance. However, the relation between diabetes and arrhythmic events has not been thoroughly investigated.

The prevalence of ventricular arrhythmias is low in subjects with no overt cardiac disease, and is greatest in patients who have an underlying arrhythmic sub- 
strate, as a result of severe myocardial damage and scarring in the setting of ischaemic heart disease and congestive heart failure (CHF) [7, 8]. This is especially true for high-grade, life-threatening, ventricular arrhythmias such as ventricular tachycardia $[7,8]$.

Therefore, to study differences in arrhythmic events between subjects with and without diabetes, it is necessary to select patients with overt cardiac disease and a high frequency of arrhythmias. We studied the relationship between diabetes status and atrial and ventricular arrhythmic events in patients with severe CHF.

\section{Subjects and methods}

The study group was derived from the PRECEDENT (Prospective Randomized Evaluation of Cardiac Ectopy with Dobutamine or Nesiritide Therapy) study. The study was a randomized, multicentre trial comparing the proarrhythmic and chronotropic effects of nesiritide (human B-type natriuretic peptide) to dobutamine in patients with symptomatic, decompensated CHF for whom inpatient parenteral vasoactive therapy was considered appropriate. Eligible patients included subjects who were over 18 years of age and had a previous history of New York Heart Association class III or IV heart failure. Exclusion criteria included recent myocardial infarction $(<48 \mathrm{~h})$, ongoing unstable angina, cardiogenic shock or prolonged hypotension, a stroke within the past month, valvular aortic stenosis, obstructive cardiomyopathy, or constrictive pericarditis. The investigational review committees of all participating study sites approved the study protocol, and informed consent was obtained from all patients.

As part of the study protocol, 24-h ambulatory electrocardiographic recordings were obtained in all patients prior to randomization to treatment with either dobutamine or nesiritide. All clinical information and Holter tapes were submitted by individual investigators to the coordinating centre for data entry and Holter analysis. Prespecified analysis of the Holter data was defined before the initiation of the study, and this report consists of all patients in whom technically adequate Holter recordings were available.

Holter monitoring. Three-channel (V1, V5, and aVF) recordings were obtained and analysed on a commercially available scanner (model 2010, Zymed Medical Instruments, Camarillo, Calif., USA) by the core laboratory. Ectopic beats were quantified by creating templates of normally conducted QRS and complexes considered to be ventricular ectopic complexes that were shown to and classified by an operator. Supraventricular ectopic complexes were those with QRS morphology matching the template of normally conducted complexes, detected strictly by their prematurity in the cardiac cycle.

Recordings were analysed for the occurrence and hourly frequency of arrhythmic events, and measures of arrhythmic activity were calculated. Two measures of atrial ectopy included the average hourly total premature atrial beats (PACs) and the number of runs of atrial tachycardia (three or more consecutive atrial premature complexes with mean R-to-R cycle length $<600 \mathrm{~ms}$ ) per $24 \mathrm{~h}$. Four measures of ventricular arrhythmic burden were prospectively defined: (i) average hourly number of single ventricular ectopic beats (PVCs), (ii) hourly paired ventricular beats, (iii) hourly repetitive ventricular beats, (iv) episodes of ventricular tachycardia per $24 \mathrm{~h}$ (defined as three or more consecutive ventricular ectopic beats with mean R-to-R cycle length $<600 \mathrm{~ms}$ ).
Definition of diabetes. Information about diabetes status was systematically obtained as part of the recruitment and follow up procedure of the PRECEDENT study. Patients were classified as having diabetes on the basis of history, regardless of duration of disease or need for antidiabetic medications. The diagnosis could have been based on a previous physician telling patients that they had diabetes (in principle, based on fasting, nonstressed blood sugar level $>126 \mathrm{mg} / \mathrm{dl}$ on at least two occasions, although this cannot be reasonably verified) or taking or having previously taken oral antihyperglycaemic medications or insulin or receiving diet therapy. All other patients who did not fulfil these criteria were included in the non-diabetic group.

Statistical analysis. The baseline characteristics of the groups were compared by Student's $t$ test for continuous variables and by the $\chi^{2}$ statistic for noncontinuous variables. Comparisons of arrhythmic events between the two groups were made using the Mann-Whitney Rank Sum test. Univariate and multivariate linear regression analyses were conducted to detect independent predictors of arrhythmic events. Factors associated with at least marginal $(p<0.15)$ univariate predictive value were entered into multiple regression models using measures of atrial or ventricular arrhythmias as dependent variables. As the distribution of continuous Holter variables was skewed, the natural $\log$ transformation was applied before statistical analyses were carried out as described by other studies $[9,10]$. In addition, a logistic regression model was used to test the association of diabetes with the presence of episodes of ventricular tachycardia. Data are shown as means \pm SEM. A $p$ value of less than 0.05 for two-sided comparisons was considered significant. All statistical analyses were carried out using the StatView statistical software (SAS Institute Inc, Cary, N.C., USA).

\section{Results}

A total of 255 patients were enrolled at 46 clinical sites. Technical failure and excess artifacts resulted in the exclusion of 19 patients (7\%) from analysis due to inadequate Holter recordings. An additional 29 patients $(11 \%)$ were excluded, because they were on amiodarone therapy. The remaining 207 patients (mean age of $61 \pm 14$ years [range 21 to 93]) constituted the study group.

Patients with diabetes had a higher incidence of ischaemic heart disease as their primary aetiology for heart failure and a lower incidence of idiopathic dilated cardiomyopathy. In addition, patients with diabetes had more renal impairment. Concomitant medications were not different between the two groups with the exception that a larger proportion of patients with diabetes was treated with vasodilators other than angiotensin converting-enzyme inhibitors (Table 1).

Effect of diabetes on atrial and ventricular ectopy. No differences in the amount of atrial ectopy between the two groups were found. The mean hourly PACs $(35 \pm 10$ vs $31 \pm 9, p=0.93)$ and the number of atrial tachycardia runs per $24 \mathrm{~h}(5.7 \pm 1.8$ vs $10 \pm 4.8, p=0.90)$ were similar in patients with and without diabetes. 
Table 1. Baseline clinical characteristics

\begin{tabular}{lcc}
\hline Characteristics & $\begin{array}{l}\text { Non-diabetic } \\
\text { patients } \\
(n=107)\end{array}$ & $\begin{array}{l}\text { Diabetic } \\
\text { patients } \\
(n=100)\end{array}$ \\
\hline Age (years) & $59 \pm 16$ & $63 \pm 12$ \\
Sex (male/female) & $72 / 35$ & $62 / 38$ \\
Primary aetiology of heart failure & \\
Ischaemic & $46(43)$ & $62(62)^{\mathrm{c}}$ \\
Idiopathic & $32(30)$ & $16(16)^{\mathrm{d}}$ \\
Hypertensive & $12(11)$ & $14(14)$ \\
Valvular or rheumatic & $7(7)$ & $2(2)$ \\
Other & $10(9)$ & $6(6)$
\end{tabular}

New York Heart Association functional class

$\begin{array}{lcc}\text { Class III } & 76(71) & 74(74) \\ \text { Class IV } & 31(29) & 26(26) \\ & & \\ \text { Laboratory data } & & \\ \text { Serum sodium (mmol/l) } & 138 \pm 4 & 138 \pm 5 \\ \text { Serum potassium (mmol/l) } & 4.1 \pm 0.5 & 4.3 \pm 0.5 \\ \text { BUN (mmol/l) } & 10.0 \pm 6.4 & 14.3 \pm 8.6 \\ \text { Creatinine ( } \mu \text { mol/l) } & 115 \pm 62 & 150 \pm 80^{f} \\ & & \\ \text { Cardiac medications } & & \\ \text { Digoxin } & 80(75) & 79(79) \\ \text { ACE inhibitors or ARB } & 85(79) & 83(83) \\ \beta \text {-Blockers } & 17(16) & 26(26) \\ \text { Other vasodilating drugs }{ }^{\mathrm{b}} & 10(9) & 21(21)^{\mathrm{g}}\end{array}$

a Other aetiologic groups included myocarditis, alcoholic cardiomyopathy, and postpartum cardiomyopathy

b Other vasodilating drugs included calcium channel blockers, nitrates, hydralazine, or $\alpha 1$-blockers

${ }^{\mathrm{c}} p=0.006$

$\mathrm{d} p=0.02$

e $p<0.0001$

f $p=0.003$

${ }^{\mathrm{g}} p=0.02$

Numbers in parentheses are percent of the group. ACE, Angiotensin converting enzyme; ARB, Angiotensin II receptor blockers

All measures of ventricular ectopy were much lower in patients with diabetes compared to patients without diabetes. The average hourly total premature ventricular beats were $16 \%$ lower in patients with diabetes $(182 \pm 27$ vs $153 \pm 28, p<0.05)$. The frequency of ventricular pairs, the mean hourly repetitive ventricular beats, and the frequency of ventricular tachycardia episodes per $24 \mathrm{~h}$ were $44 \%, 47 \%$, and $66 \%$ lower in patients with diabetes, respectively (Fig. 1).

Univariate and multivariate analysis. The effect of diabetes on ventricular arrhythmic events was examined using univariate and multivariate regression models. Possible confounding clinical and historical variables included age, sex, body weight, primary aetiology of heart failure stratified as ischaemic or nonischaemic, history of either ventricular fibrillation, sudden cardi-

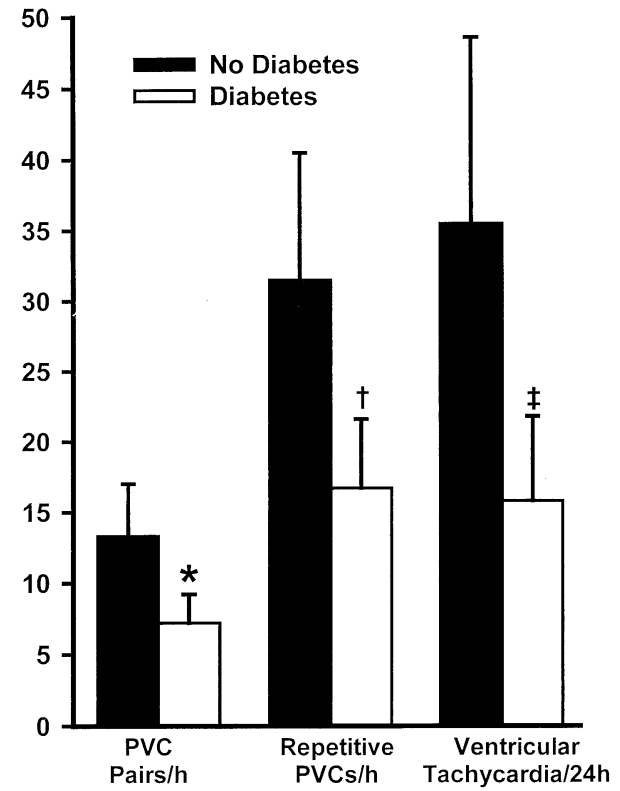

Fig. 1. Bar graph of measures of complex ventricular ectopy in patients with (open bars) and without (filled bars) diabetes. Pairs/h, average hourly ventricular pairs; Repetitive PVCs, average hourly repetitive PVCs; ventricular tachycardia/24 h, number of ventricular tachycardia episodes per 24 hours. $* p=0.03,{ }^{\dagger} p=0.01,{ }^{\ddagger} p=0.005$

ac death or automatic defibrillator implantation, New York Heart Association functional class, plasma potassium, plasma sodium, serum creatinine, and blood pressure. In addition, drug therapies (digoxin, angiotensin converting-enzyme inhibitors, $\beta$-blockers) were considered in the model.

Univariate analysis showed a negative association between diabetes and all four measures of ventricular ectopy (Table 2). Adjustment for baseline covariates had very little effect on the association between diabetes and measures of ventricular arrhythmia, indicating that these variables do not explain the negative association between diabetes and ventricular arrhythmic events.

Multivariate regression analysis also identified the female gender and higher plasma potassium levels as negative independent correlates of all measures of ventricular ectopy. Increased age was positively associated with all measures of ventricular ectopy. The univariate and multivariate relationship is shown between these variables and the frequency of ventricular tachycardia episodes (Table 3). Ischaemic heart disease was not a univariate predictor of any measure of atrial or ventricular ectopy, nor did it alter the multivariate models.

Risk of ventricular tachycardia. The effect of diabetes on the risk of developing at least one episode of ventricular tachycardia during heart failure decompensation was also calculated by simple and multiple logistic regression analysis, using the presence or absence 
Table 2. Univariate and multivariate linear model of the relationship between diabetes and the occurrence of ventricular ectopy

\begin{tabular}{|c|c|c|c|c|}
\hline \multirow[t]{2}{*}{ Ventricular ectopy measures } & \multicolumn{2}{|l|}{ Unadjusted } & \multicolumn{2}{|c|}{ Adjusted for clinical variables and drug therapies } \\
\hline & $\beta$-Coefficient (SE) & $p$ value & $\beta$-Coefficient (SE) & $p$ value \\
\hline Ventricular pairs/h & $-0.73(0.33)$ & 0.028 & $-0.70(0.32)$ & 0.030 \\
\hline Repetitive PVCs/h & $-0.50(0.23)$ & 0.027 & $-0.46(0.22)$ & 0.037 \\
\hline Ventricular tachycardia/24 h & $-0.54(0.22)$ & 0.015 & $-0.47(0.22)$ & 0.030 \\
\hline
\end{tabular}

a Clinical variables (age, sex, body weight, diabetes status, primary etiology of heart failure stratified as ischaemic or nonischaemic, history of either ventricular fibrillation, sudden cardiac death or AICD implantation, New York Heart Association functional class, serum potassium, serum creatinine, blood pressure) and drugs (digoxin, angiotensin converting-enzyme inhibitors, and $\beta$-blockers)

Table 3. Univariate and multivariate linear model of the relationship between other clinical variables and ventricular tachycardia

\begin{tabular}{|c|c|c|c|c|}
\hline & \multicolumn{2}{|l|}{ Unadjusted } & \multicolumn{2}{|c|}{ Adjusted for clinical variables and drug therapies a } \\
\hline & $\beta$-Coeff (SE) & $p$ value & $\beta$-Coeff (SE) & $p$ value \\
\hline Sex & $-0.50(0.23)$ & 0.03 & $-0.69(0.22)$ & 0.002 \\
\hline Plasma potassium & $-0.68(0.23)$ & 0.004 & $-0.56(0.22)$ & 0.01 \\
\hline
\end{tabular}

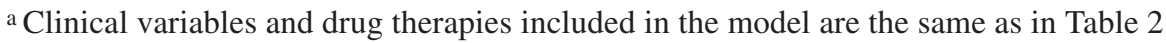

Table 4. Risk of developing an episode of ventricular tachycardia in patients with diabetes according to a multiple logistic regression model

\begin{tabular}{llll}
\hline & Odds ratio & $95 \%$ CI & $p$ value \\
\hline Unadjusted risk & 0.39 & $0.22-0.70$ & 0.001 \\
Adjusted risk $^{\mathrm{a}}$ & 0.41 & $0.22-0.75$ & 0.004 \\
\hline
\end{tabular}

a Clinical variables and drug therapies included in the model are the same as in Table 2

of at least one episode of ventricular tachycardia as the dependent variable. Variables included as independent risk factor candidates for the model were the same ones as in the multivariate analysis. In univariate logistic regression, diabetes was negatively related to the development of ventricular tachycardia (Table 4). After adjustment for all other important covariables, diabetes remained a negative predictor of a lower incidence of ventricular tachycardia (Table 4).

\section{Discussion}

The results of the study suggest that patients with diabetes and severe decompensated heart failure are less likely to develop ventricular arrhythmias compared to patients without diabetes. Despite the multiple factors that could promote ventricular ectopy in the setting of heart failure, multivariate regression analysis indicat- ed a negative independent relationship between diabetes and measures of ventricular arrhythmic events. The relative protection conferred by diabetes was especially striking for high-grade ventricular arrhythmias.

Given that patients with diabetes had more comorbidities, with a higher incidence of ischaemic heart disease and impaired kidney function, these findings are surprising. However they are in accord with a recent report indicating that patients with diabetes and severe left ventricular dysfunction had an adjusted risk for arrhythmic death almost half that of patients without diabetes as well as a substantially lower risk of arrhythmias occurring during hospitalization [11].

Diabetes and arrhythmic events. Several studies have linked diabetes to an increased risk of sudden cardiac death $[3,12]$. The association between diabetes and sudden death seems to be highest in patients with diabetic autonomic neuropathy $[13,14,15,16]$. These observations have led to the suggestion that ventricular arrhythmias are the mechanism of unexplained death in diabetic patients and that autonomic neuropathy increases the risk for arrhythmic events $[17,18$, 19, 20, 21].

Predisposition to ventricular arrhythmias has been proposed based on the association of diabetic autonomic neuropathy and QT prolongation $[19,21]$. The observation that diabetic autonomic neuropathy is associated with a substantial diminution of parasympathetic tone led to the hypothesis that relative predomi- 
nance of sympathetic activity might predispose patients with diabetes to arrhythmias $[17,18]$. However, diabetic autonomic neuropathy is associated with both parasympathetic and sympathetic denervation $[22,23$, 24]. Indeed, evaluation of muscle sympathetic nerve activity [23], and myocardial m-[123I]iodobenzylguanidine (MIBG) scintigraphy [24] indicate that severe derangements of sympathetic activity and adrenergic cardiac innervation occur concomitantly with parasympathetic dysfunction in diabetic autonomic neuropathy. Therefore, although the protective vagal activity is reduced in patients with diabetes, the absolute sympathetic tone is also substantially diminished. Consequently, the existence and clinical significance of a 'relative' predominance of sympathetic activity in patients with diabetic autonomic neuropathy remains unclear. In fact, the attenuation of sympathetic responses in the setting of heart failure [25] could be protective with regard to arrhythmias.

Although sudden deaths are often considered arrhythmic, a high rate of acute coronary events in this setting has been reported. In fact, autopsy studies reported findings consistent with acute coronary events including fresh thrombus, recent myocardial infarction, or plaque rupture in $57 \%$ to $73 \%$ of patients who died suddenly [26, 27].

In the context of sudden death in patients with diabetes, it should be emphasized, however, that currently there is no evidence that patients with diabetes (with or without autonomic neuropathy) have an increased incidence of ventricular arrhythmic events, or that the occurrence of sudden unexplained deaths in these individuals is the result of malignant ventricular arrhythmias.

The results of our study show that diabetic patients are less prone to ventricular arrhythmias. This finding argues against a propensity to arrhythmic events as the mechanism that underlies unexplained death in patients with diabetes. Although lower frequency of ventricular arrhythmias was shown in a specific cohort of diabetic patients with cardiac decompensation, it is not likely that the propensity to ventricular arrhythmias should be considerably different in patients with diabetes with milder forms of cardiac disease or no heart disease.

Possible mechanisms for the protective diabetes effect. Ventricular arrhythmia is a multifactorial event, preconditioned by structural abnormalities and modulated by several triggering mechanisms including ischaemia, metabolic and haemodynamic alterations, neurohormonal and autonomic factors [7, 8, 28, 29].

Therefore, three major factors could account for the findings of this study. Firstly, diabetes-related differences in arrhythmic substrate could have been present due to differences in underlying cardiac diagnoses, or due to myocardial abnormalities unique to the diabetic state [30]. Although ischaemic heart disease was more frequent as the primary aetiology of heart failure in patients with diabetes, ischaemic heart disease had no discernible effect on the severity of all measures of ventricular ectopy in univariate and multivariate analyses. In addition, all patients in our study had advanced heart failure as part of the inclusion criteria, suggesting that a major difference in arrhythmic substrate is not likely.

Secondly, true diabetes-distinctive differences in the electrophysiological properties of the myocardium could exist. For example, animal studies have shown that the diabetic myocardium might be less susceptible to arrhythmias in the setting of ischaemia [31, 32] and reperfusion [33].

Thirdly, diabetes-based differences in triggering events could explain our findings. Heart failure entails complex autonomic and hormonal responses including profound abnormalities in autonomic control, characterized by sympathetic overactivity and parasympathetic withdrawal [34], together with activation of endogenous vasoconstrictor systems (e.g. renin-angiotensin, norepinephrine) [29, 35]. These humoral and neural alterations might exert direct or indirect (e.g. parasympathetic withdrawal) proarrhythmic effects on the heart. In the setting of decompensated heart failure, the magnitude of neurohormonal responses, autonomic dysregulation, or end-organ sensitivity to various neurohumoral modulators could be especially important in assessing the propensity to arrhythmias.

Attenuated neurohumoral and sympathetic activation of a variety of stressors has been described in patients with diabetes, and might confer protection from arrhythmias during heart failure decompensation. For example, patients with diabetes show a diminished adrenergic response to hypoglycaemia [36, 37], exercise [38], and psychological stress [39]. Furthermore, patients with diabetes and heart failure seem to have a blunted sympathetic activation compared with patients without diabetes [25].

Our study results suggest that diabetes confers a protective effect with regard to the occurrence of ventricular arrhythmias in the setting of decompensated heart failure. Although the mechanism of action cannot be determined from these data, an endogenous mechanism of cardioprotection could be operating in these patients. The precise mechanisms of this phenomenon, however, require further investigation.

\section{References}

1. American Diabetes association (1993) Consensus Statement: role of cardiovascular risk factors in prevention and treatment of macrovascular disease in diabetes. Diabetes Care 16: 72-78

2. Grundy SM, Benjamin IJ, Burke GL et al. (1999) Diabetes and cardiovascular disease: a statement for healthcare professionals from the American Heart Association. Circulation 100: 1134-1146 
3. Curb JD, Rodriguez BL, Burchfiel CM, Abbott RD, Chiu D, Yano K (1995) Sudden death, impaired glucose tolerance, and diabetes in Japanese American men. Circulation 91: 2591-2595

4. Toyry JP, Niskanen LK, Mantysaari MJ, Lansimies EA, Uusitupa MI (1996) Occurrence, predictors, and clinical significance of autonomic neuropathy in NIDDM. Ten-year follow-up from the diagnosis. Diabetes 45: 308-315

5. Bernardi L, Ricordi L, Lazzari P et al. (1992) Impaired circadian modulation of sympathovagal activity in diabetes. A possible explanation for altered temporal onset of cardiovascular disease. Circulation 86: 1443-1452

6. Spallone V, Bernardi L, Ricordi L et al. (1993) Relationship between the circadian rhythms of blood pressure and sympathovagal balance in diabetic autonomic neuropathy. Diabetes 42: 1745-1752

7. Akhtar M, Garan H, Lehmann MH, Troup PJ (1991) Sudden cardiac death: management of high-risk patients. Ann Intern Med 114: 499-512

8. Zipes DP, Wellens HJ (1998) Sudden cardiac death. Circulation 98: 2334-2351

9. Sami M, Kraemer H, Harrison DC, Houston N, Shimasaki C, DeBusk RF (1980) A new method for evaluating antiarrhythmic drug efficacy. Circulation 62: 1172-1179

10. Teerlink JR, Jalaluddin M, Anderson S et al. (2000) Ambulatory ventricular arrhythmias in patients with heart failure do not specifically predict an increased risk of sudden death. PROMISE (Prospective Randomized Milrinone Survival Evaluation) Investigators. Circulation 101: 40-46

11. Whang W, Bigger JT Jr (2000) Diabetes and outcomes of coronary artery bypass graft surgery in patients with severe left ventricular dysfunction: results from The CABG Patch Trial database. The CABG Patch Trial Investigators and Coordinators. J Am Coll Cardiol 36: 1166-1172

12. Balkau B, Jouven X, Ducimetiere P, Eschwege E (1999) Diabetes as a risk factor for sudden death. Lancet 354: 1968-1969

13. Ewing DJ, Campbell IW, Clarke BF (1976) Mortality in diabetic autonomic neuropathy. Lancet i: 601-603

14. O'Brien IA, McFadden JP, Corrall RJ (1991) The influence of autonomic neuropathy on mortality in insulin-dependent diabetes. Q J Med 79: 495-502

15. Lloyd-Mostyn RH, Watkins PJ (1975) Defective innervation of heart in diabetic autonomic neuropathy. BMJ 3: 15-17

16. Page MM, Watkins PJ (1978) Cardiorespiratory arrest and diabetic autonomic neuropathy. Lancet i: $14-16$

17. Bernardi L, Ricordi L, Lazzari P et al. (1992) Impaired circadian modulation of sympathovagal activity in diabetes. A possible explanation for altered temporal onset of cardiovascular disease. Circulation 86: 1443-1452

18. Weston PJ, Gill GV (1999) Is undetected autonomic dysfunction responsible for sudden death in Type 1 diabetes mellitus? The 'dead in bed' syndrome revisited. Diabet Med 16: 626-631

19. Ewing DJ, Boland O, Neilson JM, Cho CG, Clarke BF (1991) Autonomic neuropathy, QT interval lengthening, and unexpected deaths in male diabetic patients. Diabetologia 34: 182-185

20. Stevens MJ, Raffel DM, Allman KC et al. (1998) Cardiac sympathetic dysinnervation in diabetes: implications for enhanced cardiovascular risk. Circulation 98: 961-968

21. Bellavere F, Ferri M, Guarini L et al. (1988) Prolonged QT period in diabetic autonomic neuropathy: a possible role in sudden cardiac death? Br Heart J 59: 379-383
22. Bellavere F, Balzani I, De Masi G et al. (1992) Power spectral analysis of heart-rate variations improves assessment of diabetic cardiac autonomic neuropathy. Diabetes 41: 633-640

23. Hoffman RP, Sinkey CA, Kienzle MG, Anderson EA (1993) Muscle sympathetic nerve activity is reduced in IDDM before overt autonomic neuropathy. Diabetes 42 : 375-380

24. Kreiner G, Wolzt M, Fasching P et al. (1995) Myocardial $\mathrm{m}-[123 \mathrm{I}]$ iodobenzylguanidine scintigraphy for the assessment of adrenergic cardiac innervation in patients with IDDM. Comparison with cardiovascular reflex tests and relationship to left ventricular function. Diabetes 44: 543549

25. Burger AJ, Aronson D (2001) Blunted sympathetic response in diabetic patients with decompensated congestive heart failure. Int J Cardiol 81: 243-249

26. Davies MJ (1992) Anatomic features in victims of sudden coronary death. Coronary artery pathology. Circulation 85 : I19-I24

27. Farb A, Tang AL, Burke AP, Sessums L, Liang Y, Virmani R (1995) Sudden coronary death. Frequency of active coronary lesions, inactive coronary lesions, and myocardial infarction. Circulation 92: 1701-1709

28. Podrid PJ, Fogel RI, Fuchs TT (1992) Ventricular arrhythmia in congestive heart failure. Am J Cardiol 69: 82G-95G (discussion 95G-96G)

29. Packer M (1992) The neurohormonal hypothesis: a theory to explain the mechanism of disease progression in heart failure [editorial]. J Am Coll Cardiol 20: 248-254

30. Zarich SW, Nesto RW (1989) Diabetic cardiomyopathy. Am Heart J 118: 1000-1012

31. Ravingerova T, Neckar J, Kolar F et al. (2001) Ventricular arrhythmias following coronary artery occlusion in rats: is the diabetic heart less or more sensitive to ischaemia? Basic Res Cardiol 96: 160-168

32. Ravingerova T, Stetka R, Volkovova K et al. (2000) Acute diabetes modulates response to ischemia in isolated rat heart. Mol Cell Biochem 210: 143-151

33. Kusama Y, Hearse DJ, Avkiran M (1992) Diabetes and susceptibility to reperfusion-induced ventricular arrhythmias. J Mol Cell Cardiol 24: 411-421

34. Floras JS (1993) Clinical aspects of sympathetic activation and parasympathetic withdrawal in heart failure. J Am Coll Cardiol 22: 72A-84A

35. Bigger JT (1987) Why patients with congestive heart failure die: arrhythmias and sudden cardiac death. Circulation 75: IV28-IV35

36. Hoeldtke RD, Boden G, Shuman CR, Owen OE (1982) Reduced epinephrine secretion and hypoglycemia unawareness in diabetic autonomic neuropathy. Ann Intern Med 96: 459-462

37. Hoffman RP, Singer-Granick C, Drash AL, Becker DJ (1991) Plasma catecholamine responses to hypoglycemia in children and adolescents with IDDM. Diabetes Care 14: 81-88

38. Bottini P, Tantucci C, Scionti L et al. (1995) Cardiovascular response to exercise in diabetic patients: influence of autonomic neuropathy of different severity. Diabetologia 38: $244-250$

39. Forst T, Pfutzner A, Jahn C et al. (1996) Decreased sympatho-adrenal activity in diabetic patients with autonomic dysfunction following mental stress. J Auton Nerv Syst 61: $31-36$ 